\title{
Malignant Melanoma of the Vulva and Vagina: A US Population-Based Study of 1863 Patients
}

\author{
Christoph Wohlmuth ${ }^{1,2,3} \cdot$ Iris Wohlmuth-Wieser ${ }^{4}$ Taymaa May $^{1,2} \cdot$ Danielle Vicus $^{2,5} \cdot$ Lilian T. Gien $^{2,5}$. \\ Stéphane Laframboise ${ }^{1,2}$
}

Published online: 29 November 2019

(c) The Author(s) 2019

\begin{abstract}
Background Vulvar melanoma (VuM) and vaginal melanoma (VaM) represent a unique subgroup of malignant melanomas with important differences in biology and treatment.

Objective The objective of this study was to describe the epidemiology and prognosis of VuM and VaM in a large representative cohort.

Methods Women with invasive VuM or VaM were identified from the Surveillance, Epidemiology and End Results-18 population representing $27.8 \%$ of the US population. Data on age, ethnicity, stage, location, histopathology, primary surgery, and lymphadenectomy were collected. The Kaplan-Meier method was used to analyze disease-specific and overall survival. Univariate and multivariate regression models were used to identify factors with a significant association with disease-specific survival.

Results A total of $1400 \mathrm{VuM}$ and $463 \mathrm{VaM}$ were included for further analysis; $78.6 \%$ and $49.7 \%$ of women with VuM and VaM underwent surgery, but only $52.9 \%$ of women with non-metastatic VuM and $42.9 \%$ of women with non-metastatic VaM undergoing surgery had lymph node assessment; one third of these had positive nodes. Superficial spreading was the most common subtype in VuM, and nodular melanoma in VaM $(p<0.001)$. The median disease-specific survival was 99 months (95\% confidence interval 60-138) and 19 months (95\% confidence interval 16-22), respectively. Survival was significantly associated with age at diagnosis, ethnicity, stage, surgery, lymph node metastases, histologic subtype, ulceration, mitotic count, and tumor thickness in VuM, and stage, surgery, and lymph node involvement in VaM. In the Cox model, lymph node status and number of mitoses remained independent predictors of outcome in $\mathrm{VuM}$; in $\mathrm{VaM}$, only lymph node status remained significant. Conclusions The overall prognosis of VuM and VaM remains poor. The American Joint Committee on Cancer staging system is applicable and should be used for VuM; however, lymph node status and mitotic rate are the most important predictors of survival. Lymph node status should be assessed and patients with positive nodes may be candidates for adjuvant treatment.
\end{abstract}

Christoph Wohlmuth

christoph.wohlmuth@outlook.com

1 Division of Gynecologic Oncology, Department of Surgical Oncology, University Health Network, Toronto, ON, Canada

2 Department of Obstetrics and Gynecology, University of Toronto, Toronto, ON, Canada

3 Department of Obstetrics and Gynecology, Paracelsus Medical University Salzburg, Müllner Hauptstrasse 48, 5020 Salzburg, Austria

4 Department of Dermatology, Paracelsus Medical University, Salzburg, Austria

5 Division of Gynecologic Oncology, Odette Cancer Centre, Sunnybrook Health Sciences Centre, Toronto, ON, Canada

\section{Key Points}

The American Joint Committee on Cancer staging system used for cutaneous melanoma is applicable for vulvar melanoma and predicts outcome.

Lymph node involvement is the most important predictor of survival.

Mitotic rate is an important predictor and should be reported separately because it is not part of the most recent American Joint Committee on Cancer staging system.

Vulvar and vaginal melanoma differ significantly in terms of biology and vaginal melanoma is associated with worse prognosis. 


\section{Introduction}

Primary malignant melanoma of the vulva (VuM) and vagina (VaM) represent an important subgroup of malignancies with significant differences in terms of biology and treatment compared with the more common and welldescribed squamous cell carcinoma [1]. Important differences also exist in terms of anatomic considerations and surgical approach compared with other melanomas [2-5]. Traditionally, VuM and VaM were categorized as mucosal melanoma, but this has recently been questioned by studies examining molecular characteristics of these lesions, which showed that melanomas of the female genital tract differ in terms of mutational characteristics from mucosal and cutaneous melanomas. It has therefore been suggested that VuM and VaM represent a unique subclass [6-8].

The literature on female genital melanoma is scarce; to date, there is only one prospective study following 71 women with $\mathrm{VuM}$ who underwent radical (hemi-)vulvectomy [9]. Retrospective series suggest that the prognosis and survival are significantly worse compared with cutaneous melanoma [10-14].

Staging for VuM has been extrapolated from cutaneous melanoma and the American Joint Committee on Cancer (AJCC) system is now being used instead of the International Federation of Gynecology and Obstetrics classification, although it remains unclear whether this accurately reflects the behavior of VuM [15]. While surgery remains the primary treatment modality, the US Food and Drug Administration approval of checkpoint inhibitors and targeted therapy has drastically changed the medical management of advanced and metastatic melanoma and significantly improved overall- and melanoma-specific survival [16-22]. Based on these recent advances in skin melanoma and the poor overall prognosis of genital melanomas reported in smaller series, comprehensive study of $\mathrm{VuM}$ and $\mathrm{VaM}$ is warranted. The aim of this study is to describe the epidemiologic, clinical, and histopathologic characteristics of VuM and VaM and to analyze their impact on survival in a large representative cohort.

\section{Methods}

\subsection{Study Population}

The Surveillance, Epidemiology and End Results (SEER) database, a registry funded by the National Cancer Institute, was used to identify cases of VuM and VaM. The SEER-18 population (including Atlanta, Connecticut, Detroit, Hawaii, Iowa, New Mexico, San Francisco-Oakland, Seattle-Puget Sound, Utah, Los Angeles, San Jose-Monterey, Rural
Georgia, the Alaska Native Tumor Registry, Greater California, Greater Georgia, Kentucky, Louisiana and New Jersey) in its November 2018 submission version (1975-2016) was used [23].

Patients with a diagnosis of invasive VuM and VaM were identified in the SEER*Stat 8.3.5 database and their clinical data were retrieved; cases of in situ melanoma were not included. Only patients with known age and listing in the research database were included.

Cases that did not meet the International Association of Cancer Registries criteria were excluded from further analyses. Patients' age at diagnosis, year of diagnosis, ethnicity, SEER stage (i.e., localized, regional, distant disease), location of melanoma (labia majora, labia minora, clitoris, overlapping), histopathology, and type of primary surgery and lymphadenectomy were collected. The AJCC stage was collected from all cases where information was available and included both AJCC sixth and seventh editions. Histologic subtypes were identified using the following International Classification of Diseases for Oncology, Third Revision codes: 8720, 8721, 8722, 8723, 8726, 8730, 8740, 8741, 8743, 8744, 8745, 8746, 8761, 8770, 8771, 8772, 8773, and 8774 . Data on surgery were grouped as local, radical, debulking, and surgery not otherwise specified. Cases where only a biopsy or local destructive procedure (i.e., cryosurgery, laser) was performed were labeled as "no surgery performed".

Information on ulceration (present, absent, unknown), mitotic count (mitoses $/ \mathrm{mm}^{2}$ ), and tumor thickness (mm) was extracted. Data on mitotic count were further grouped and analyzed as follows; "0", "1", "2-10", and "> 10", according to the proposed categories by Nagarajan et al. [24]. Vital status, disease-specific survival, and time from diagnosis to last follow-up or death were collected. Patients who were reported to have died from a different malignancy were excluded from disease-specific survival analyses. The use of SEER data is exempt from ethics board approval and all retrieved data excluded personal identifiers.

\subsection{Statistics}

Descriptive statistics was used to report demographic data. Continuous variables were compared using the Student $t$ test, Mann-Whitney test, or Wilcoxon test, as appropriate. More than two groups were compared using analysis of variance or the Kruskal-Wallis test. Cross-tables and the Chi-square test were used to compare categorical data. The Kaplan-Meier method with log-rank test was used to analyze disease-specific survival for both vulvar and vaginal primary sites. Survival was calculated from the date of diagnosis to the date of death. Univariate analysis was used to identify factors with a significant association with disease-specific survival. These were incorporated into a multivariate, stepwise-forward, Cox 
proportional hazards regression model to detect covariates that were independently related to disease-specific survival. Statistical analysis was performed using SPSS Version 25 (IBM, Armonk, NY, USA). A $p$ value $\leq 0.05$ was considered statistically significant.

\section{Results}

\subsection{Patient Characteristics}

A total of 1910 women with VuM or VaM were identified in the SEER-18 population meeting the above described criteria; 47 cases were excluded from further analysis because they did not meet the International Association of Cancer Registries criteria and were metastases, recurrences, or extensions from a different primary tumor. There were 1863 cases included for further analysis comprising $1400 \mathrm{VuM}$ and $463 \mathrm{VaM}$. This represents $1.0 \%(1863 / 177,807)$ of all malignant melanomas in women, $5.3 \%(1400 / 26,250)$ of all vulvar malignancies, and 5.5\% (463/8409) of all vaginal malignancies in the SEER-18 population.

Patient demographics differed significantly between VuM and VaM (Table 1). The median age difference was 3 years and women with $\mathrm{VuM}$ were diagnosed at a younger age compared with women with VaM; $14.7 \%$ and $28.3 \%$ of women with VuM and VaM were non-white, respectively $(p<0.001)$. Significantly more cases of VaM were diagnosed in advanced-stage disease (i.e., regional or distant) as compared with VuM $(46.5 \%$ vs. $31.6 \%, p<0.001)$. The AJCC stages were reported in 634 cases of VuM and were distributed as follows: stage I: $34.9 \%$, stage II: $34.4 \%$, stage III:

Table 1 Patient characteristics

\begin{tabular}{|c|c|c|c|}
\hline Characteristic & Vulva $(n=1400)$ & Vagina $(n=463)$ & $P$ value \\
\hline \multicolumn{4}{|l|}{ Age at diagnosis, years } \\
\hline Mean age \pm SD & $65.6 \pm 18.2$ & $69.5 \pm 14.3$ & $<0.001$ \\
\hline Median age (range) & $68.0(10-107)$ & $71.0(21-100)$ & 0.002 \\
\hline \multicolumn{4}{|l|}{ Ethnicity, $n(\%)$} \\
\hline White & $1194(85.3)$ & $332(71.7)$ & \multirow[t]{6}{*}{$<0.001$} \\
\hline African American & $49(3.5)$ & $44(9.5)$ & \\
\hline Hispanic & $102(7.3)$ & $33(7.1)$ & \\
\hline Asian or Pacific Islander & $41(2.9)$ & $53(11.4)$ & \\
\hline American Indian or Alaska Native & $4(0.3)$ & $1(0.2)$ & \\
\hline Unknown & $10(0.7)$ & $0(0.0)$ & \\
\hline \multicolumn{4}{|l|}{ SEER stage, $n(\%)$} \\
\hline Localized & $736(52.6)$ & $170(36.7)$ & \multirow[t]{4}{*}{$<0.001$} \\
\hline Regional & 349 (24.9) & 99 (21.4) & \\
\hline Distant & $94(6.7)$ & $116(25.1)$ & \\
\hline Unstaged & $221(15.8)$ & $78(16.8)$ & \\
\hline \multicolumn{4}{|l|}{ Surgery of primary melanoma, $n(\%)$} \\
\hline Performed & 1101 (78.6) & $230(49.7)$ & \multirow[t]{7}{*}{$<0.001$} \\
\hline Local & $707(64.2)$ & $109(47.4)$ & \\
\hline Radical & 208 (18.9) & $77(33.5)$ & \\
\hline Debulking & $8(0.7)$ & $3(1.3)$ & \\
\hline Not specified & $178(16.2)$ & $41(17.8)$ & \\
\hline No surgery performed ${ }^{\mathrm{a}}$ & $284(20.3)$ & $218(47.1)$ & \\
\hline Not reported & $15(1.1)$ & $15(3.2)$ & \\
\hline \multicolumn{4}{|l|}{ Lymphadenectomy, $n(\%)$} \\
\hline Performed & $654(46.7)$ & $128(27.6)$ & \multirow[t]{6}{*}{$<0.001$} \\
\hline Nodal metastases & $223(34.1)$ & $39(30.5)$ & \\
\hline No nodal metastases & $427(65.3)$ & $89(69.5)$ & \\
\hline Result not reported & $4(0.6)$ & $0(0.0)$ & \\
\hline Not performed & $547(39.1)$ & $273(59.0)$ & \\
\hline Not reported & 199 (14.2) & $62(13.4)$ & \\
\hline
\end{tabular}

$S D$ standard deviation, SEER Surveillance Epidemiology and End Results Program stage (localized, regional, distant)

${ }^{\mathrm{a}}$ Including all patients where only biopsies and/or local destructive procedures were performed 
$24.0 \%$, and stage IV: $6.8 \%$; VaM was only staged as local, regional, and distant. No surgery was performed in $16.2 \%$ of women with $\mathrm{VuM}$ and these included 16 cases with local destructive procedures, whereas $47.1 \%$ of women with VaM did not undergo surgery, including eight cases with local destructive procedures $(p<0.001)$. Only 564/1067 (52.9\%) of women with non-metastatic VuM undergoing surgery and $79 / 184(42.9 \%)$ of women with non-metastatic VaM undergoing surgery had lymph node assessment (full lymphadenectomy or removal of sentinel lymph node). In both VuM and VaM, approximately one third of the cases had lymph node involvement on final pathology (Table 1).

\subsection{Histopathology}

The mean size of the primary tumor was $31.1 \pm 44.1 \mathrm{~mm}$ for VuM and $41.9 \pm 46.7 \mathrm{~mm}$ for VaM, respectively $(p<0.001)$. The histopathologic details are shown in Table 2. Almost half of the VuM were classified as superficial spreading, whereas the majority of women with VaM had a nodular subtype. The distribution of histologic subtypes is shown in Fig. 1. Breslow thickness was grouped into four categories and $31.6 \%$ had ultra-thin melanomas, defined as a tumor

Table 2 Histologic characteristics

\begin{tabular}{|c|c|c|c|}
\hline Characteristics & Vulva & Vagina & $P$ value \\
\hline \multicolumn{4}{|l|}{ Histologic subtype, $n(\%)$} \\
\hline Superficial spreading & $346(48.4)$ & $5(5.4)$ & $<0.001$ \\
\hline Nodular & $248(34.7)$ & $51(55.4)$ & \\
\hline Lentiginous & $48(6.7)$ & $8(8.7)$ & \\
\hline Spindle cell & $36(5.0)$ & $12(13.0)$ & \\
\hline Amelanotic & $15(2.1)$ & $6(6.5)$ & \\
\hline Epithelioid & $6(0.8)$ & $2(2.2)$ & \\
\hline $\begin{array}{l}\text { Mixed epithelioid and } \\
\text { spindle cell }\end{array}$ & $10(1.4)$ & $5(5.4)$ & \\
\hline Other & $6(0.8)$ & $3(3.3)$ & \\
\hline \multicolumn{4}{|l|}{ Ulceration, $n(\%)$} \\
\hline Present & $396(55.5)$ & $\mathrm{n} / \mathrm{a}$ & $\mathrm{n} / \mathrm{a}$ \\
\hline Absent & $317(44.5)$ & $\mathrm{n} / \mathrm{a}$ & \\
\hline \multicolumn{4}{|c|}{ Mitotic count (mitoses $\left./ \mathrm{mm}^{2}\right), n(\%)$} \\
\hline 0 & $61(17.8)$ & $\mathrm{n} / \mathrm{a}$ & $\mathrm{n} / \mathrm{a}$ \\
\hline 1 & $48(14.0)$ & $\mathrm{n} / \mathrm{a}$ & \\
\hline $2-10$ & $163(47.5)$ & $\mathrm{n} / \mathrm{a}$ & \\
\hline$>10$ & $71(20.7)$ & $\mathrm{n} / \mathrm{a}$ & \\
\hline \multicolumn{4}{|c|}{ Tumor thickness (mm), $n(\%)$} \\
\hline$\leq 1.00$ & $215(31.6)$ & $\mathrm{n} / \mathrm{a}$ & $\mathrm{n} / \mathrm{a}$ \\
\hline $1.01-2.00$ & $148(21.7)$ & $\mathrm{n} / \mathrm{a}$ & \\
\hline $2.01-4.00$ & $108(15.9)$ & $\mathrm{n} / \mathrm{a}$ & \\
\hline$>4.00$ & $210(30.8)$ & $\mathrm{n} / \mathrm{a}$ & \\
\hline
\end{tabular}

$n / a$ not applicable thickness $<1 \mathrm{~mm} ; 30.8 \%$ had a Breslow thickness $>4 \mathrm{~mm}$. Ulcerations were found in approximately half of the VuM.

\subsection{Outcome}

The median overall survival was 53 months (95\% confidence interval (CI) 46-60 months) and 16 months (95\% CI 14-18 months); the median disease-specific survival was 99 months (95\% CI 60-138 months) and 19 months (95\% CI 16-22 months) for women with VuM and VaM, respectively. A total of 664 patients with VuM and 320 patients with VaM died from melanoma. Figure 2 compares the incidence (Fig. 2a) and 2-year survival rate (Fig. 2b) of skin, mucosal, and vulvovaginal melanomas over time and illustrates the drastic increase in incidence of cutaneous melanomas while vulvovaginal melanomas remained relatively stable. Although the 2-year survival rate for skin melanoma has improved over time, no concordant improvement has been observed for vulvovaginal melanoma.

The disease-specific survival by clinical, histopathological, and treatment characteristics is shown in Table 3 and the corresponding Kaplan-Meier curves are shown in Figs. 3, 4 and 5. Age at diagnosis, ethnicity, SEER and AJCC stage, surgery, lymph node metastases, histologic subtype, ulceration, mitotic count, and tumor thickness were significantly associated with survival outcomes in VuM. Disease-specific survival was associated with SEER stage, surgery, and lymph node involvement in VaM. In the Cox proportional hazard model, lymph node status and number of mitoses remained independent predictors of outcome in VuM (Table 4), whereas in VaM only lymph node status was significantly associated with survival outcome (Table 4).

\section{Discussion}

Vulvar and vaginal melanomas represent rare malignancies of the female genital tract [13]. The current evidence and management strategies are mainly based on smaller retrospective series and extrapolation from cutaneous melanoma [2, 10-13]. In this study, we used the SEER-18 registry representing $27.8 \%$ of the US population [23] to characterize demographic, clinical, and histopathologic features in VuM and VaM and assess their implication on prognosis.

We have shown that the overall survival in VuM and VaM is worse compared with the survival rate in cutaneous melanoma across all stages (Fig. 2). This is in agreement with smaller retrospective series from the MD Anderson Cancer Center, Memorial Sloan Kettering Cancer Center, and the AGO Germany and may be explained by later diagnosis and different biology [10-12]. The survival of women with VaM was especially poor. While more women diagnosed with VaM already had metastatic 
Vulva

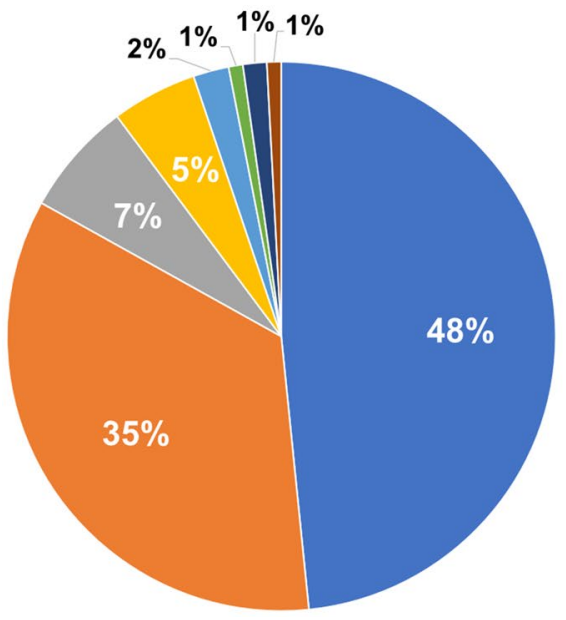

Vagina

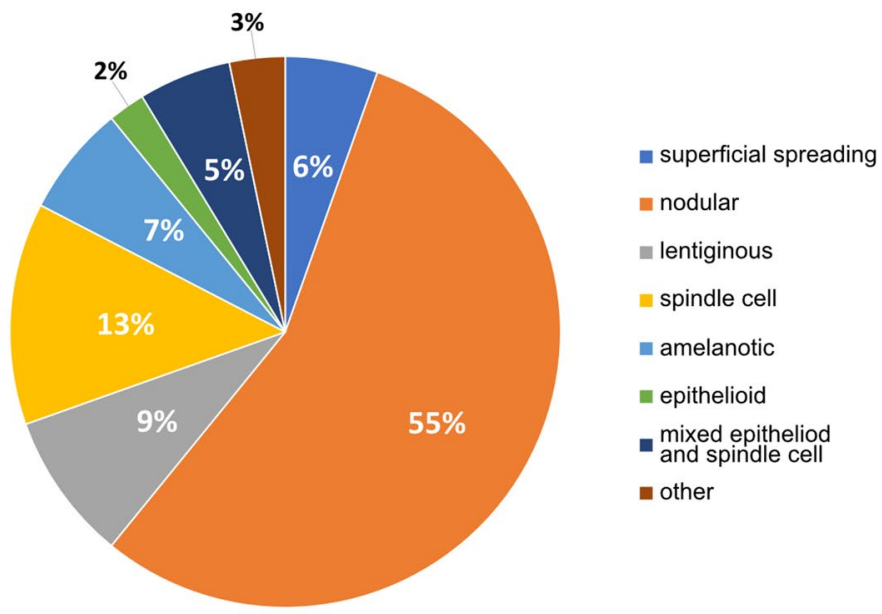

Fig. 1 Histologic subtypes of vulvar and vaginal melanoma

Fig. 2 Change of melanoma incidence and survival over time. a Annual incidence of skin, mucosal, and vulvovaginal melanoma per $1,000,000$ persons in the Surveillance, Epidemiology and End Results (SEER)-9 population. b Twoyear overall survival rate of skin, mucosal, and vulvovaginal melanoma in the SEER-9 population
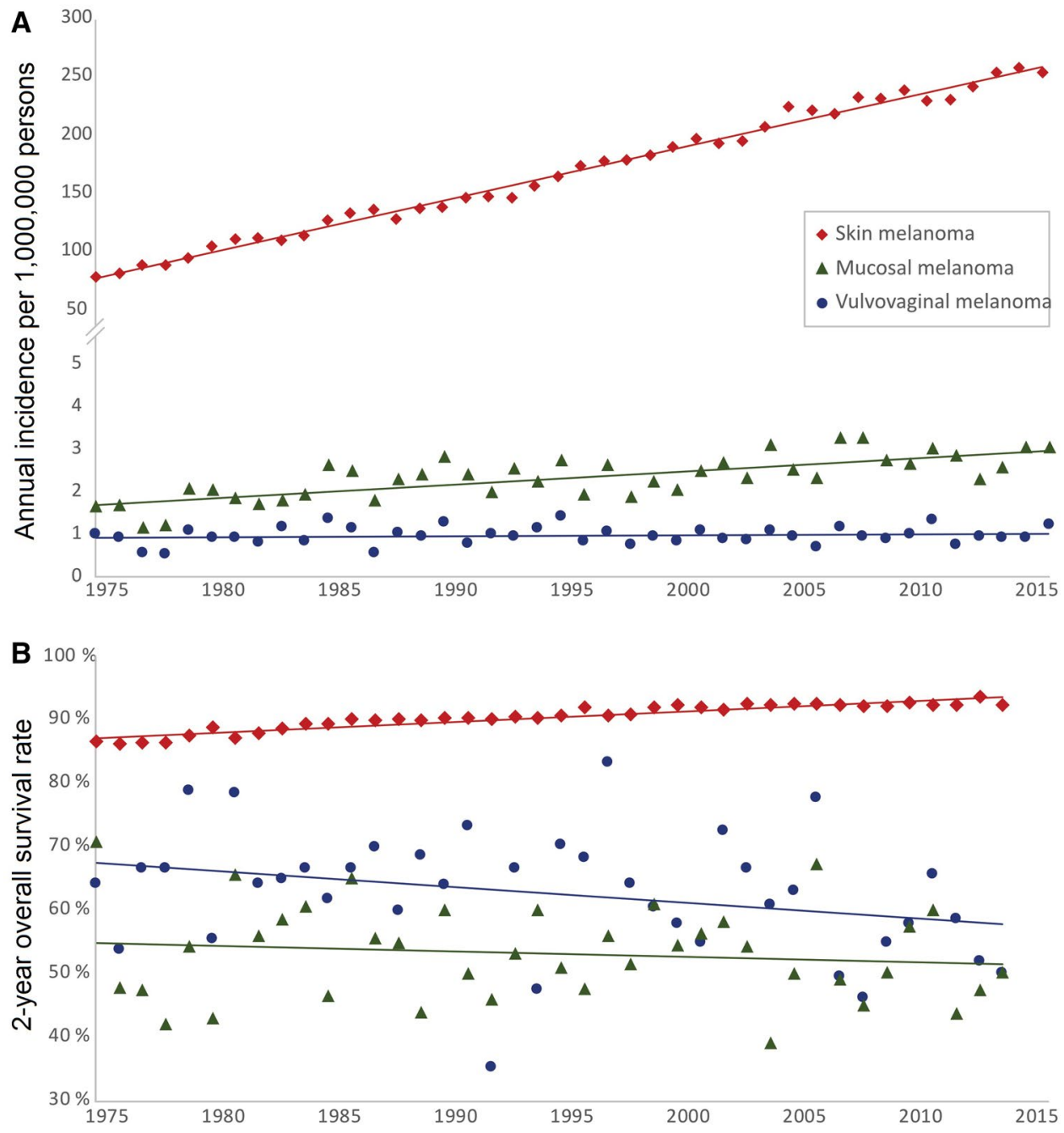
Table 3 Five-year disease-specific survival rate by clinical, histological, and treatment characteristics

\begin{tabular}{|c|c|c|c|c|}
\hline \multirow[t]{2}{*}{ Parameter } & \multicolumn{2}{|l|}{ Vulva } & \multicolumn{2}{|l|}{ Vagina } \\
\hline & $\% \pm \mathrm{SE}$ & $P$ value & $\% \pm \mathrm{SE}$ & $P$ value \\
\hline Age, years & & $<0.001$ & & 0.079 \\
\hline$\leq$ Median age & $\leq 68: 67.6 \pm 2.1$ & & $\leq 71: 20.6 \pm 3.1$ & \\
\hline$>$ Median age & $>68: 42.3 \pm 2.5$ & & $>71: 12.8 \pm 3.2$ & \\
\hline Ethnicity & & $<0.001$ & & 0.628 \\
\hline White & $58.7 \pm 1.8$ & & $17.8 \pm 2.7$ & \\
\hline African American & $33.2 \pm 8.1$ & & $16.6 \pm 8.2$ & \\
\hline Hispanic & $52.7 \pm 6.3$ & & $9.3 \pm 7.9$ & \\
\hline Asian or Pacific Islander & $34.0 \pm 10.0$ & & $18.9 \pm 7.0$ & \\
\hline American Indian or Alaska Native & 0 & & 0 & \\
\hline Unknown & $80.0 \pm 17.9$ & & $\mathrm{n} / \mathrm{a}$ & \\
\hline Year of diagnosis & & 0.736 & & 0.254 \\
\hline 1975-1986 & $61.1 \pm 4.2$ & & $21.5 \pm 7.6$ & \\
\hline 1987-1996 & $57.0 \pm 4.2$ & & $13.2 \pm 4.9$ & \\
\hline 1997-2006 & $55.9 \pm 2.8$ & & $22.5 \pm 4.3$ & \\
\hline 2007-2016 & $55.2 \pm 2.9$ & & $13.9 \pm 3.5$ & \\
\hline SEER stage & & $<0.001$ & & $<0.001$ \\
\hline Localized & $70.6 \pm 2.1$ & & $23.3 \pm 4.0$ & \\
\hline Regional & $35.4 \pm 3.2$ & & $21.2 \pm 5.4$ & \\
\hline Distant & $13.4 \pm 4.9$ & & $6.3 \pm 2.7$ & \\
\hline Unstaged & $59.7 \pm 4.4$ & & $17.9 \pm 6.6$ & \\
\hline AJCC stage & & $<0.001$ & & $\mathrm{n} / \mathrm{a}$ \\
\hline I & $83.6 \pm 3.1$ & & $\mathrm{n} / \mathrm{a}$ & \\
\hline II & $51.8 \pm 4.7$ & & $\mathrm{n} / \mathrm{a}$ & \\
\hline III & $24.9 \pm 5.2$ & & $\mathrm{n} / \mathrm{a}$ & \\
\hline IV & $6.4 \pm 6.0$ & & $\mathrm{n} / \mathrm{a}$ & \\
\hline Unstaged & $57.3 \pm 2.2$ & & $\mathrm{n} / \mathrm{a}$ & \\
\hline Surgery & & $<0.001$ & & 0.007 \\
\hline Local & $61.6 \pm 2.3$ & & $21.1 \pm 4.6$ & \\
\hline Radical & $43.5 \pm 4.4$ & & $22.5 \pm 6.0$ & \\
\hline Debulking & $66.7 \pm 27.2$ & & 0 & \\
\hline Surgery not specified & $63.1 \pm 4.1$ & & $23.6 \pm 8.2$ & \\
\hline No surgery performed & $49.0 \pm 3.8$ & & $11.5 \pm 3.1$ & \\
\hline Not reported & $33.3 \pm 15.7$ & & $14.3 \pm 12.8$ & \\
\hline Lymph node metastases & & $<0.001$ & & 0.003 \\
\hline Negative nodes & $70.8 \pm 2.8$ & & $27.1 \pm 5.4$ & \\
\hline One positive node & $29.0 \pm 5.7$ & & $10.7 \pm 6.5$ & \\
\hline Two or more positive nodes & $20.8 \pm 5.2$ & & 0 & \\
\hline Not assessed & $57.7 \pm 2.2$ & & $15.5 \pm 2.7$ & \\
\hline Histologic subtype & & $<0.001$ & & 0.833 \\
\hline Superficial spreading & $72.0 \pm 3.0$ & & $33.3 \pm 27.2$ & \\
\hline Nodular & $40.9 \pm 4.1$ & & $20.9 \pm 6.5$ & \\
\hline Lentiginous & $64.8 \pm 8.7$ & & 0 & \\
\hline Spindle cell & $57.3 \pm 10.1$ & & $11.1 \pm 10.5$ & \\
\hline Amelanotic & $24.4 \pm 13.9$ & & $25.0 \pm 21.7$ & \\
\hline Epithelioid & $80.0 \pm 17.9$ & & 0 & \\
\hline Mixed epithelioid and spindle cell & $17.1 \pm 15.6$ & & 0 & \\
\hline Other & $\mathrm{n} / \mathrm{a}$ & & $33.3 \pm 27.2$ & \\
\hline Unknown & $54.3 \pm 2.4$ & & $16.9 \pm 2.6$ & \\
\hline
\end{tabular}


Table 3 (continued)

\begin{tabular}{|c|c|c|c|c|}
\hline \multirow[t]{2}{*}{ Parameter } & \multicolumn{2}{|l|}{ Vulva } & \multicolumn{2}{|l|}{ Vagina } \\
\hline & $\% \pm \mathrm{SE}$ & $P$ value & $\% \pm \mathrm{SE}$ & $P$ value \\
\hline Ulceration & & $<0.001$ & & $\mathrm{n} / \mathrm{a}$ \\
\hline Present & $73.9 \pm 3.4$ & & $\mathrm{n} / \mathrm{a}$ & \\
\hline Absent & $40.2 \pm 3.6$ & & $\mathrm{n} / \mathrm{a}$ & \\
\hline Not reported & $57.3 \pm 2.2$ & & $\mathrm{n} / \mathrm{a}$ & \\
\hline Mitotic count (mitoses $/ \mathrm{mm}^{2}$ ) & & 0.001 & & $\mathrm{n} / \mathrm{a}$ \\
\hline 0 & $75.4 \pm 8.6$ & & $\mathrm{n} / \mathrm{a}$ & \\
\hline 1 & $73.4 \pm 9.8$ & & $\mathrm{n} / \mathrm{a}$ & \\
\hline $2-10$ & $48.6 \pm 6.9$ & & $\mathrm{n} / \mathrm{a}$ & \\
\hline$>10$ & $31.4 \pm 9.0$ & & $\mathrm{n} / \mathrm{a}$ & \\
\hline Unknown & $56.6 \pm 1.8$ & & $\mathrm{n} / \mathrm{a}$ & \\
\hline Tumor thickness (mm) & & $<0.001$ & & $\mathrm{n} / \mathrm{a}$ \\
\hline$\leq 1.00$ & $74.5 \pm 3.9$ & & $\mathrm{n} / \mathrm{a}$ & \\
\hline $1.01-2.00$ & $61.7 \pm 5.1$ & & $\mathrm{n} / \mathrm{a}$ & \\
\hline $2.01-4.00$ & $58.7 \pm 6.9$ & & $\mathrm{n} / \mathrm{a}$ & \\
\hline$>4.00$ & $32.5 \pm 5.0$ & & $\mathrm{n} / \mathrm{a}$ & \\
\hline Unknown & $55.7 \pm 2.2$ & & $\mathrm{n} / \mathrm{a}$ & \\
\hline
\end{tabular}

AJCC American Joint Committee on Cancer, SE standard error, SEER Surveillance Epidemiology and End Results Program stage (localized, regional, distant)

disease (25.1\% vs. $6.7 \%)$, survival was consistently worse across all disease stages compared with $\mathrm{VuM}$ and therefore the poor prognosis cannot solely be attributed to a later diagnosis in more advanced stages of the disease (Figs. 3b, 5a).

The histopathologic subtypes differed significantly between VuM and VaM with the superficial spreading type being the most common form in VuM. This subtype is generally associated with a better prognosis [25]. In contrast, the nodular subtype was found in more than half of the VaM and has previously been associated with worse survival $[25,26]$.

Consistent with previous findings from the GOG-73 study, where 71 women with $\mathrm{VuM}$ were prospectively observed [9], the AJCC staging system was prognostic of disease-specific survival (Fig. 3b) and can be used in women with VuM. However, in our multivariate Cox model, lymph node status was the most important independent predictor of survival (Table 4; Fig. 3d).

The staging system is currently in its eighth edition and includes Breslow thickness, ulceration, lymph node involvement, and distant metastases. A recent retrospective study from the MD Anderson Cancer Center suggested that dermal mitotic rate is an important independent predictor of overall and disease-specific survival in VuM [24]. While the previous AJCC edition included mitotic rate in its T-stage, this has been omitted in the current version [15]. We have validated the findings from this study [24] in a larger cohort and have shown that mitotic rate is an independent predictor of survival that remained significant in the multivariate analysis; we therefore recommend routine assessment during a pathologic work-up.

Surgery remains the primary treatment modality for all locally resectable melanomas [2, 4, 5]. A surgical margin of $0.5-1.0 \mathrm{~cm}$ for melanoma in situ, $1 \mathrm{~cm}$ for invasive melanoma with a Breslow thickness $\leq 1 \mathrm{~mm}, 1-2 \mathrm{~cm}$ for a Breslow thickness of 1.01-2 mm, and $2 \mathrm{~cm}$ for a Breslow thickness of $\geq 2.01 \mathrm{~mm}$ is generally recommended [2, $4,5]$. While this may be easily achievable without major functional disturbances in most parts of the body, it can be challenging for VuM and VaM in terms of preservation of continence and sexual function. However, strategies of more radical surgery have been attempted in the past in view of the poor prognosis of genital melanoma [9]. While prospective data are lacking, retrospective data indicate that there is no benefit to more radical surgical approaches compared to local procedures with the above-mentioned surgical margins [10-12]. This is consistent with the findings in the present study where radical surgery did not have a better outcome (Fig. 3c). In fact, the disease-specific survival was worse, but this is likely attributable to the fact that more radical procedures were performed in cases with advanced disease.

Because of the lack of prospective data, the role of lymph node assessment has been controversial in the past and only $52.9 \%$ of women with non-metastatic VuM and $42.9 \%$ of women with non-metastatic VaM in the SEER-18 population did undergo a lymphadenectomy or a sentinel-node biopsy. In this study, however, the lymph 
A

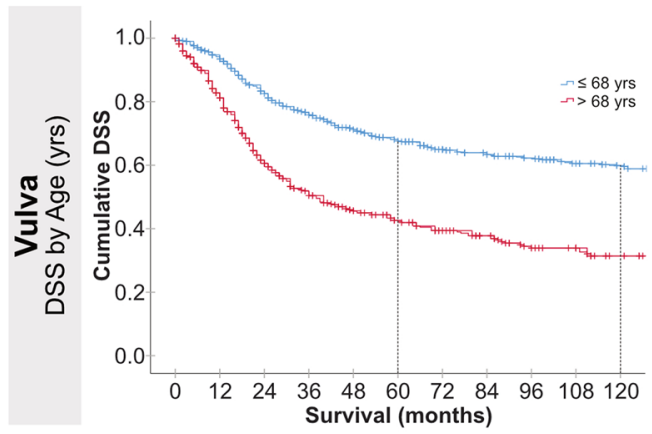

C

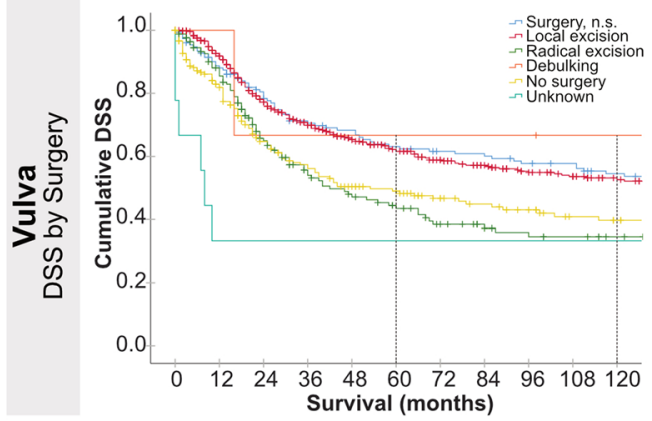

$\mathbf{E}$

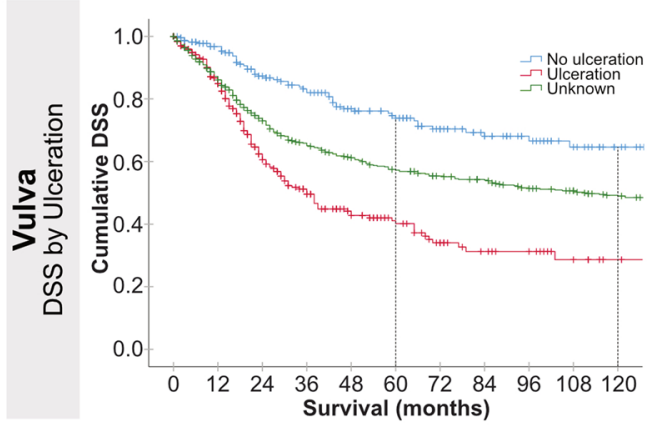

Fig. 3 Kaplan-Meier plots for 5- and 10-year disease-specific survival (DSS) for women with vulvar melanoma. a DSS by age. b DSS by American Joint Committee on Cancer (AJCC) stage. c DSS by
B

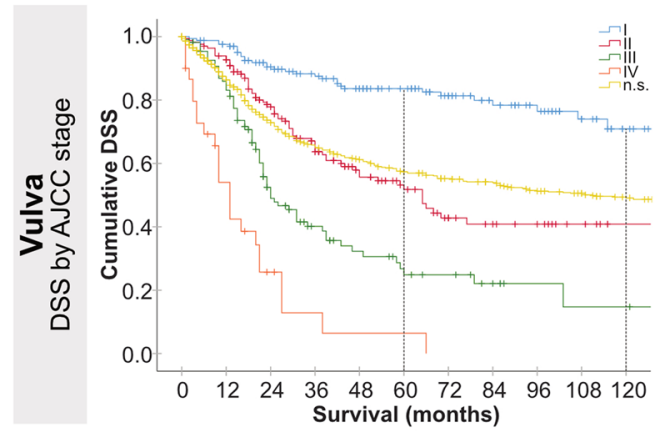

D

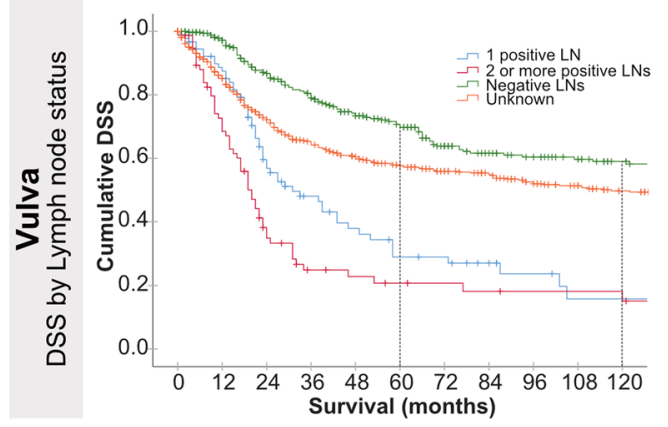

surgery. d DSS by lymph node status. E DSS by ulceration. $n$.s. not specified, SEER Surveillance Epidemiology and End Results Program stage (localized, regional, distant), yrs years

A

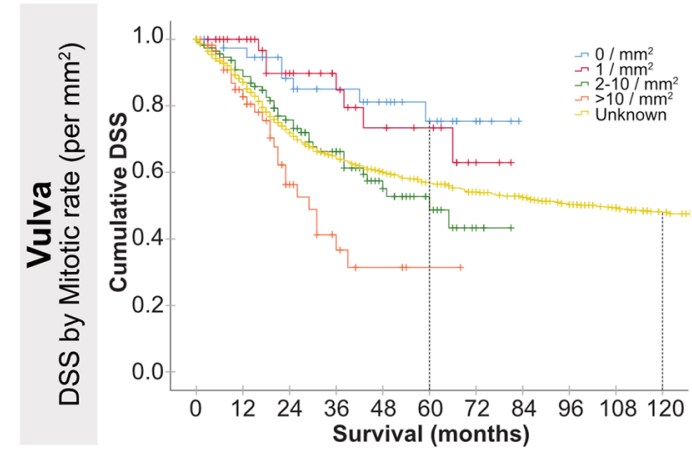

Fig. 4 Kaplan-Meier plots for 5- and 10-year disease-specific survival (DSS) for histopathologic characteristics in women with vulvar melanoma. a DSS by mitotic rate. b DSS by tumor thickness. AJCC

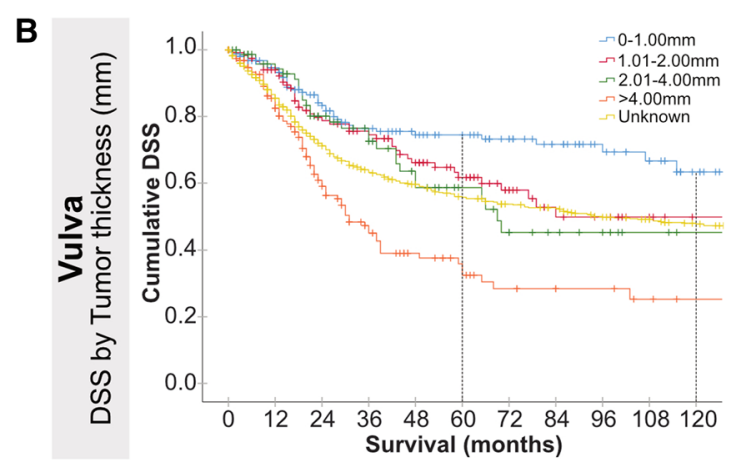

American Joint Committee on Cancer, n.s. not specified, SEER Surveillance Epidemiology and End Results Program stage (localized, regional, distant), yrs years 
A

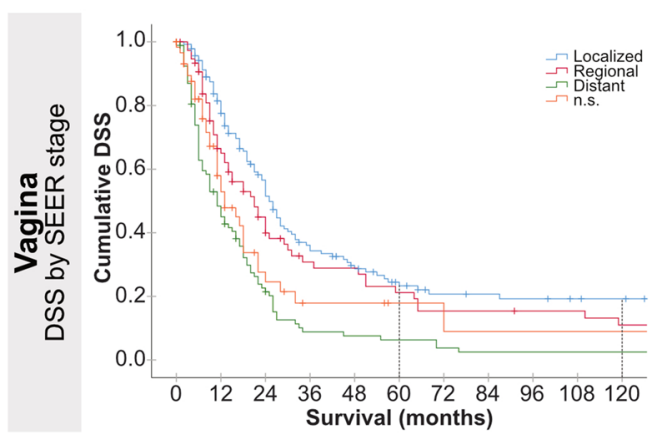

C

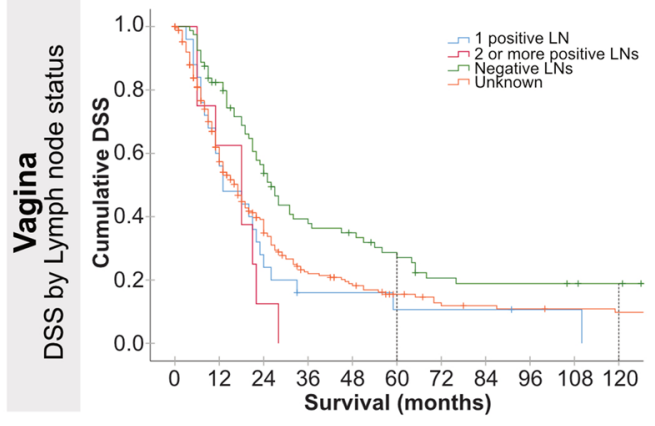

Fig. 5 Kaplan-Meier plots for 5- and 10-year disease-specific survival (DSS) women with vaginal melanoma. a DSS by Surveillance Epidemiology and End Results (SEER) Program stage (localized,

B

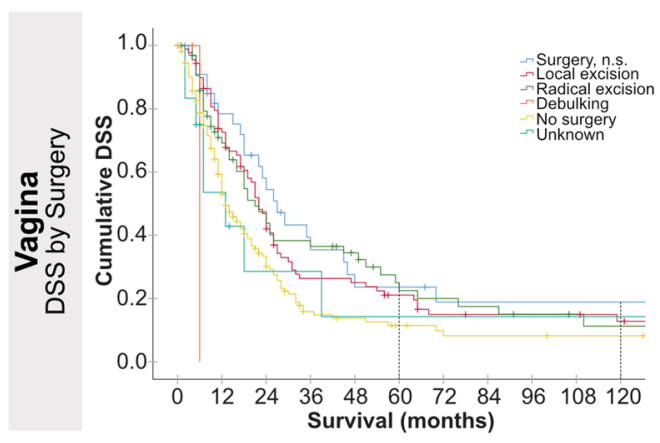

regional, distant) stage. b DSS by surgery. c DSS by lymph node status. AJCC American Joint Committee on Cancer, n.s. not specified, yrs years

Table 4 Multivariate Cox proportional hazard model for vulvar and vaginal melanoma

\begin{tabular}{|c|c|c|c|c|}
\hline \multirow[t]{2}{*}{ Prognostic factor } & \multicolumn{2}{|l|}{ Vulva } & \multicolumn{2}{|l|}{ Vagina } \\
\hline & Hazard ratio $(95 \% \mathrm{CI})$ & $P$ value & Hazard ratio $(95 \% \mathrm{CI})$ & $P$ value \\
\hline Lymph node metastases & & $<0.001$ & & 0.004 \\
\hline Negative nodes & Reference & & Reference & \\
\hline One positive node & $3.148(1.538-6.445)$ & 0.002 & $1.710(1.003-2.916)$ & 0.049 \\
\hline Two or more positive nodes & $4.432(2.087-9.408)$ & $<0.001$ & $3.432(1.514-7.778)$ & 0.003 \\
\hline Mitoses (per mm², cont.) & $1.111(1.019-1.212)$ & 0.017 & - & \\
\hline
\end{tabular}

CI confidence interval, cont. continuous variable

node status was the most important independent predictor of survival and lymph node involvement was consistently associated with prognosis in previous prospective and retrospective analyses [9-12]. The EORTC 18071, Checkmate-238, and Keynote-054 studies have shown improved survival in surgically resected stage III melanoma treated with adjuvant checkpoint inhibitors. Therefore, ipilimumab, nivolumab, and pembrolizumab have recently been approved for adjuvant treatment [27-29]. This underlines the need for all women with malignant melanoma of the female genital tract $>1 \mathrm{~mm}$ depth of invasion to undergo sentinel-node biopsy [3]. In thin melanomas $\leq 1 \mathrm{~mm}$, other risk features including mitotic rate should be evaluated and a sentinel-node biopsy may be offered in those with higher risk features [3]. In the MSLT-II trial, immediate completion lymph-node dissection did not increase melanoma-specific survival among patients with sentinel-node metastases and can therefore be omitted [30]. Although the above-mentioned study protocols allowed inclusion of mucosal and vulvovaginal melanoma, the results have not been separately analyzed or reported. However, preliminary data from pooled subgroup analyses from several randomized clinical trials on cytotoxic T-lymphocyte-associated antigen-4 (CTLA-4) and programmed death-ligand 1 (PD-L1) inhibitors indicate similar survival improvements compared to cutaneous melanoma albeit to a lesser extent [21]. 


\section{Strengths and Limitations}

This study investigates a large series of well-described cases of VuM and VaM and is representative of the North American population. Because of the rarity of female genital melanomas, prospective data are scarce. Similarly, previously published retrospective single-center experiences have been limited by the small number precluding firm conclusions. The study is, however, limited by its retrospective design and the use of registry data, which do not allow confirmation and preclude central pathology review. In addition, margin status of the surgical specimens was not available, limiting firm conclusions regarding the extent of surgery. Information on chemotherapy and radiation is limited in the SEER database and has therefore not been included in this study.

\section{Conclusions}

In summary, $\mathrm{VuM}$ and $\mathrm{VaM}$ represent a unique subclass of malignant melanomas with poor prognosis. The AJCC staging system is applicable for $\mathrm{VuM}$, but lymph node status and mitotic rate are the most important predictors for disease-specific survival. Because the latter is not included in the current AJCC staging system, we recommend reporting it separately. Lymph node status should be assessed in all applicable patients with VuM and VaM. Those with positive lymph nodes may be candidates for adjuvant treatment.

Acknowledgements Open access funding provided by Paracelsus Medical University.

Author Contributions CW, IWW: study conception, data acquisition and analysis, interpretation of data and original draft of the manuscript, review and editing. TM, DV, LG, SL: investigation, methodology, review and editing of the manuscript.

\section{Compliance with Ethical Standards}

Funding No external funding was used in the preparation of this article.

Conflict of interest Christoph Wohlmuth, Iris Wohlmuth-Wieser, Taymaa May, Danielle Vicus, Lilian T. Gien, and Stéphane Laframboise have no conflicts of interest that are directly relevant to the content of this article.

Open Access This article is distributed under the terms of the Creative Commons Attribution-NonCommercial 4.0 International License (http://creativecommons.org/licenses/by-nc/4.0/), which permits any noncommercial use, distribution, and reproduction in any medium, provided you give appropriate credit to the original author(s) and the source, provide a link to the Creative Commons license, and indicate if changes were made.

\section{References}

1. Koh W-J, Greer BE, Abu-Rustum NR, Campos SM, Cho KR, Chon HS, et al. Vulvar cancer, version 1.2017, NCCN clinical practice guidelines in oncology. J Natl Compr Cancer Netw. 2017;15:92-120.

2. Coit DG, Thompson JA, Algazi A, Andtbacka R, Bichakjian CK, Carson WE, et al. NCCN guidelines insights: melanoma, version 3.2016. J Natl Compr Cancer Netw. 2016;14:945-58.

3. Wong SL, Faries MB, Kennedy EB, Agarwala SS, Akhurst TJ, Ariyan C, et al. Sentinel lymph node biopsy and management of regional lymph nodes in melanoma: American Society of Clinical Oncology and Society of Surgical Oncology clinical practice guideline update. J Clin Oncol. 2018;36:399-413.

4. Dummer R, Hauschild A, Lindenblatt N, Pentheroudakis G, Keilholz U. Cutaneous melanoma: ESMO clinical practice guidelines for diagnosis, treatment and follow-up. Ann Oncol. 2015;26:v126-32.

5. AWMF. S3-Leitlinie zur Diagnostik, Therapie und Nachsorge des Melanoms, V 3.1. AWMF; 2018. . https://www.awmf.org. Accessed 23 Oct 2019.

6. Hou JY, Baptiste C, Hombalegowda RB, Tergas AI, Feldman R, Jones NL, et al. Vulvar and vaginal melanoma: a unique subclass of mucosal melanoma based on a comprehensive molecular analysis of 51 cases compared with 2253 cases of nongynecologic melanoma. Cancer. 2017;123:1333-44.

7. Saglam O, Messina J, Naqvi SMH, Teer JK, Lee J, Zhang Y, et al. Female genitourinary tract melanoma: mutation analysis with clinicopathologic correlation; a single-institution experience. Melanoma Res. 2018;28:586-91.

8. Rouzbahman M, Kamel-Reid S, Al Habeeb A, Butler M, Dodge J, Laframboise $S$, et al. Malignant melanoma of vulva and vagina. J Low Genit Tract Dis. 2015;19:350-3.

9. Phillips GL, Bundy BN, Okagaki T, Kucera PR, Stehman FB. Malignant melanoma of the vulva treated by radical hemivulvectomy: a prospective study of the gynecologic oncology group. Cancer. 1994;73:2626-32.

10. Trimble EL, Lewis JL, Williams LL, Curtin JP, Chapman D, Woodruff JM, et al. Management of vulvar melanoma. Gynecol Oncol. 1992;45:254-8.

11. Verschraegen CF, Benjapibal M, Supakarapongkul W, Levy LB, Ross M, Atkinson EN, et al. Vulvar melanoma at the M. D. Anderson Cancer Center: 25 years later. Int J Gynecol Cancer. 2001;11:359-64.

12. Räber G, Mempel V, Jackisch C, Hundeiker M, Heinecke A, Kürzl $\mathrm{R}$, et al. Malignant melanoma of the vulva: report of 89 patients. Cancer. 1996;78:2353-8.

13. Pleunis N, Schuurman MS, Van Rossum MM, Bulten J, Massuger LF, De Hullu JA, et al. Rare vulvar malignancies; incidence, treatment and survival in the Netherlands. Gynecol Oncol. 2016;142:440-5.

14. Sinasac SE, Petrella TM, Rouzbahman M, Sade S, Ghazarian D, Vicus D. Melanoma of the vulva and vagina: surgical management and outcomes based on a clinicopathologic review of 68 cases. J Obstet Gynaecol Can. 2019;41:762-71.

15. Gershenwald JE, Hess KR, Thompson JF, Long GV, Ross MI, Lazar AJ, et al. Melanoma of the skin. In: AJCC cancer staging manual, 8th ed. American Joint Committee on Cancer; 2017. p. 563-88.

16. Hodi FS, O'Day SJ, McDermott DF, Weber RW, Sosman JA, Haanen JB, et al. Improved survival with ipilimumab in patients with metastatic melanoma. N Engl J Med. 2010;363:711-23. 
17. Wolchok JD, Chiarion-Sileni V, Gonzalez R, Rutkowski P, Grob J-J, Cowey CL, et al. Overall survival with combined nivolumab and ipilimumab in advanced melanoma. N Engl J Med. 2017;377:1345-56.

18. Robert C, Grange F, Mortier L, Karaszewska B, Rutkowski P, Mackiewicz A, et al. Improved overall survival in melanoma with combined dabrafenib and trametinib. N Engl J Med. 2015;372:30-9.

19. Long GV, Stroyakovskiy D, Gogas H, Levchenko E, De Braud F, Larkin J, et al. Dabrafenib and trametinib versus dabrafenib and placebo for Val600 BRAF-mutant melanoma: a multicentre, double-blind, phase 3 randomised controlled trial. Lancet. 2015;386:444-51.

20. Robert C, Schachter J, Long GV, Arance A, Grob JJ, Mortier L, et al. Pembrolizumab versus ipilimumab in advanced melanoma. N Engl J Med. 2015;372:2521-32.

21. D’Angelo SP, Larkin J, Sosman JA, Lebbé C, Brady B, Neyns B, et al. Efficacy and safety of nivolumab alone or in combination with ipilimumab in patients with mucosal melanoma: a pooled analysis. J Clin Oncol. 2017;35:226-35.

22. Hamid O, Robert C, Ribas A, Hodi FS, Walpole E, Daud A, et al. Antitumour activity of pembrolizumab in advanced mucosal melanoma: a post-hoc analysis of KEYNOTE-001, 002, 006. Br J Cancer. 2018;119:670-4.

23. Surveillance, Epidemiology, and End Results (SEER) Program SEER*Stat Database: incidence-SEER 18 Regs research data + Hurricane Katrina impacted Louisiana cases, Nov $2018 \mathrm{sub}$ (1975-2016 varying) — linked to county attributes-total. https:// seer.cancer.gov. Accessed 28 Jul 2019.
24. Nagarajan P, Curry JL, Ning J, Piao J, Torres-Cabala CA, Aung PP, et al. Tumor thickness and mitotic rate robustly predict melanoma-specific survival in patients with primary vulvar melanoma: a retrospective review of 100 cases. Clin Cancer Res. 2017;23:2093-104.

25. Lattanzi M, Lee Y, Simpson D, Moran U, Darvishian F, Kim RH, et al. Primary melanoma histologic subtype: impact on survival and response to therapy. J Natl Cancer Inst. 2019;111:186-8.

26. de Vries E, Houterman S, Janssen-Heijnen MLG, Nijsten T, van de Schans SAM, Eggermont AMM, et al. Up-to-date survival estimates and historical trends of cutaneous malignant melanoma in the south-east of The Netherlands. Ann Oncol. 2007;18:1110-6.

27. Eggermont AMM, Chiarion-Sileni V, Grob J-J, Dummer R, Wolchok JD, Schmidt H, et al. Prolonged survival in stage III melanoma with ipilimumab adjuvant therapy. N Engl J Med. 2016;375:1845-55.

28. Weber J, Mandala M, Del Vecchio M, Gogas HJ, Arance $\mathrm{AM}$, Cowey $\mathrm{CL}$, et al. Adjuvant nivolumab versus ipilimumab in resected stage III or IV melanoma. N Engl J Med. 2017;377:1824-35.

29. Eggermont AMM, Blank CU, Mandala M, Long GV, Atkinson V, Dalle S, et al. Adjuvant pembrolizumab versus placebo in resected stage III melanoma. N Engl J Med. 2018;378:1789-801.

30. Faries MB, Thompson JF, Cochran AJ, Andtbacka RH, Mozzillo N, Zager JS, et al. Completion dissection or observation for sentinel-node metastasis in melanoma. N Engl J Med. 2017;376:2211-22. 